\title{
Patient-centered Weight Tracking as an Early Cancer Detection Strategy
}

\author{
Jonathan J. Hue', Sarah C. Markt ${ }^{2}$, Goutham Rao ${ }^{3}$, Jordan M. Winter ${ }^{1}$ \\ ${ }^{1}$ Department of Surgery, Division of Surgical Oncology, University Hospitals Cleveland Medical Center, ${ }^{2}$ Department of \\ Population and Quantitative Health Sciences, Case Western Reserve University School of Medicine, ${ }^{3}$ Department of Family \\ Medicine, University Hospitals Cleveland Medical Center, OH, USA
}

\begin{abstract}
Early detection is a valued strategy to decrease cancer mortality rates; however, new strategies are needed. Unintentional weight loss (UWL) is experienced by patients across the cancer spectrum, but often goes unnoticed. Patient-centered weight tracking may be a useful early detection marker. Fifty patients were enrolled in a prospective patient-centered weight tracking trial. Patients received a scale and monetary compensation to participate. A reminder to measure and record weight was texted to participants for 26 consecutive weeks. Most patients were black (86.0\%) and female (68.0\%). The median age was 47 years (range: $22-84$ years). Many participants had Medicaid (42.0\%) and the median household income by home zip code was $\$ 31,046$. After 26 weeks, $90 \%$ of patients had recorded at least one weight. Among all patients, $73.7 \%$ of all possible weights were recorded and the median response rate per patient was $92.3 \%$ (24 of 26 weights). There was no difference in the response rates during the first and second halves of the study $(77.7 \%$ vs. $69.7 \%, P=0.53)$. The range of weight change over the study period was $16.1 \%$ loss to $25.0 \%$ gain, with $56 \%$ of patients maintaining stable weight. Seven patients $(14.0 \%)$ lost more than $5 \%$ weight and 11 patients $(22.0 \%)$ gained over $5 \%$. Of the seven patients with weight loss, two (4.0\% of the cohort) were determined to have UWL. Patient-centered weight tracking is feasible and inexpensive, and has potential as an early detector of UWL. Further studies are needed to apply this strategy to detect underlying malignancies.
\end{abstract}

Key Words Body weight, Weight loss, Early detection of cancer, Technology

\section{INTRODUCTION}

Unintentional weight loss (UWL) is typically defined as a decrease from baseline body weight of greater than five percent within a six- to twelve-month period [1]. Several retrospective studies have estimated the incidence of UWL in the general population to be somewhere between $1 \%$ to $7 \%$ per year in adults who are 60 to 80 years old [2-9]. There is a wide range of possible causes for UWL, such as depression, functional decline in diverse organ systems, and gastrointestinal diseases. The most commonly described etiology, occult malignancy, is responsible for roughly $20 \%$ of all UWL cases [1-9].

UWL has been reported in a diverse range of malignancies, including colon, lung, prostate, pancreatic, and gastric cancer [10-15]. Despite these facts, it is unclear why UWL has largely been overlooked as a potential pan-cancer biomarker. To highlight this point, a previous report demonstrat- ed that weight loss was unrecognized by clinicians in $60 \%$ of cases, and $27 \%$ of those patients with weight loss were subsequently diagnosed with a malignancy [16].

While there has been progress in cancer treatment, cancer still ranks as the second-most common cause of death in the United States, with over 600,000 cancer-related deaths annually $[17,18]$. One in five patients in this country are expected to ultimately die from cancer [19]. In the year 2020 alone, $1,800,000$ cancers will be diagnosed in the United States [1719]. Approximately one in every two males and one in three females will be diagnosed with some forms of malignancy in their lifetime [17,19,20].

Cancer is challenging to treat successfully when it presents at an advanced stage. Early cancer detection, therefore, is critical to improve prognosis. Despite the fact that over $\$ 6$ billion dollars is spent annually on cancer research by the National Cancer Institute, only $10 \%$ is allocated to early de-

Received August 18, 2020, Revised September 8, 2020, Accepted September 11, 2020

Correspondence to Jonathan J. Hue, E-mail: Jonathan.Hue@UHHospitals.org, https://orcid.org/0000-0002-1608-8874

Check for updates

(i) (5) This is an Open Access article distributed under the terms of the Creative Commons Attribution Non-Commercial License, which permits unrestricted noncommercial use, distribution, and reproduction in any medium, provided the original work is properly cited.

Copyright @ 2020 Korean Society of Cancer Prevention 
tection [21]. In the private sector, billions of dollars have been invested in development and validation of sophisticated and expensive liquid biopsies, along with other molecular and imaging-based early detection strategies [22]. While such approaches may hold promise in the future, a simpler, non-invasive, and inexpensive early detection strategy may also have utility. The goal of the present study is to determine the feasibility of weekly self-weighing in order to detect UWL in an undifferentiated primary care population in a single family medicine practice.

\section{MATERIALS AND METHODS}

\section{Patient enrollment}

Consecutive patients in a single Family Medicine practice (co-author GR) were offered trial entry starting on July 2, 2019. Patients were excluded if they declined participation, did not have access to a telephone with short message service (SMS) texting capabilities, were unable to send or receive text messages, or did not speak English. During seven clinic days over the subsequent two months, a total of 50 participants provided informed consent (out of a total of 100 patients seen, recruitment rate $=50 \%$ ). Enrolled patients were well-established in the practice of the co-author Goutham Rao, with whom he is very familiar.

Enrollment closed on August 20, 2019. Patients received a stipend for their participation. The final amount was based on the total number of weights that were recorded, with a maximum compensation of $\$ 100$ per patient ( $\$ 3.85$ for each weekly weight recording). This study was approved by the Institutional Review Board (IRB) at University Hospitals Cleveland Medical Center (IRB No. STUDY20190565).

\section{Data collection}

Patients' names, addresses, and telephone numbers were obtained and stored in a REDCap (Research Electronic Data Capture) database hosted at University Hospitals Cleveland Medical Center. Additional patient information such as age, sex, self-identified race, insurance status, medical comorbidities, and the number of active medications were also obtained from a review of patients' medical records. Hypertension and gastroesophageal reflux disease (GERD) were the most common comorbidities and therefore specifically recorded for each patient. Less common comorbidities were recorded by the organ system (pulmonary, cardiovascular, endocrine, etc.). We obtained estimates of household income by linking the patient's home zip code to income data obtained from the United States Census Bureau [23].

\section{Weight tracking}

All participants were asked to weigh themselves on a weekly basis using a standard bathroom scale provided to them as part of the study. Patients were counseled to record their weight at a similar time of day each week and wear comparable, light clothing each time to minimize variability. We partnered with Devblock Technologies to implement a simple patient-centered data collection system. Patients were sent a weekly SMS text as a reminder to record their current weight. SMS responses were automatically uploaded into a secure central data repository. Devblock digital platforms are Health Insurance Portability and Accountability Act (HIPAA) and Health Information Technology for Economic and Clinical Health Act (HITECH) compliant.

Patients were asked to measure and report their weight at weekly intervals for 26 weeks (half of a year). Updated lists of patient weights were relayed to the study investigators every two weeks. Only a single attempt was made to contact patients who had not recorded any weights after six weeks of enrollment. The work-flow of the cohort study is summarized in Figure 1.

\section{Outcomes}

The primary outcome was patient engagement, defined as the number of weekly weights recorded by each participant. Study retention was based on the number of participants who recorded at least one weight.

\section{Statistical analysis}

Descriptive statistics were used to summarize the cohort, including weight reporting. We analyzed compliance in the entire group of 50 patients as the "intent-to-study" cohort. A subgroup analysis was also performed in the 45 patients who ultimately participated as the "on-study" cohort.

As a secondary analysis, we analyzed patient weight trends. A patient's first recorded weight during the trial was used as their baseline. This weight was used as a baseline instead of a previously recorded weight in an attempt to limit variability in weight, related to the scale provided for the study. Percent weight changes from baseline were calculated for each subsequent measurement using the formula: [(cur-

\begin{tabular}{|c|c|c|}
\hline $\begin{array}{l}\text { Patient } \\
\text { consented, } \\
\text { registered, } \\
\text { and provided } \\
\text { with } \\
\text { bathroom } \\
\text { scale }\end{array}$ & $\begin{array}{l}\text { Monday, week 1: } \\
\text { "Thank you for participating in the University Hospital } \\
\text { Weight Tracking Trial. You will receive a weekly } \\
\text { reminder to enter your weight. Please respond by } \\
\text { sending your weight to the nearest kilogram }(\mathrm{kg}) \\
\text { (ex: } 75) \text {. Please try to take your weight at a consistent } \\
\text { time of day every week (ex: in the morning or before } \\
\text { bed). Please contact the trial team with any question } \\
\text { at }(x x x) x x x-x x x x . "\end{array}$ & $\begin{array}{l}\text { Monday, week 2-26: } \\
\text { "Reminder to record } \\
\text { your weight! Please } \\
\text { respond by sending } \\
\text { your weight to the } \\
\text { nearest kilogram } \\
\text { (ex: 75)." }\end{array}$ \\
\hline
\end{tabular}

Figure 1. Weight tracking feasibility study design, University Hospitals Cleveland Medical Center Primary Care Clinic, July 2019 to January 2020. 
rent weight - baseline weight) / baseline weight $\times 100 \%$ ]. Clinically significant weight loss was defined as a decrease in body weight by $\geq 5 \%$ from their first recorded weight. Weight gain was defined as an increase of $\geq 5 \%$ from their first recorded weight. Stable weight was defined as fluctuations between $5 \%$ weight loss and $5 \%$ weight gain. Patients who had significant weight loss or gain from baseline at any time point were identified. The primary aim of this study was not to detect weight loss, but rather to determine the feasibility of a home, patient-centered weight tracking strategy performed at weekly intervals.

Patients with significant weight changes were grouped into (1) those where weight trended back towards baseline (transient weight changes) and (2) those with persistent weight loss or (3) those with persistent weight gain. Subgroup analyses were also performed in patients ages 45 years or older to better understand compliance in the target demographic at greatest risk for malignancy [20]. The Wilcoxon rank-sum test was used to compare response rates between the first and second halves of the trial to assess for trial fatigue. StataSE ver. 16 (Statacorp LLC, College Station, TX, USA) was used for statistical analyses and a $P$-value less than 0.05 was used to indicate statistical significance.

Table 1. Demographic and clinical data of the participants in the weight tracking feasibility study, University Hospitals Cleveland Medical Center Primary Care Clinic, July 2019 to January $2020(n=50)$

\begin{tabular}{lc}
\hline \multicolumn{1}{c}{ Patient characteristic } & Value \\
\hline Female & $34(68.0)$ \\
Male & $16(32.0)$ \\
Race & \\
Black & $43(86.0)$ \\
White & $7(14.0)$ \\
Age $(\mathrm{yr})$ & $47(22-84)$ \\
Active medications & $6(0-21)$ \\
Insurance & $16(32.0)$ \\
Private insurance & $21(42.0)$ \\
Medicaid & $6(12.0)$ \\
Medicare & $31,046(12,800-67,451)$ \\
Median household income $(\$)^{\mathrm{a}}$ & \\
Comorbidities & $24(48.0)$ \\
Hypertension & $18(36.0)$ \\
GERD & $15(30.0)$ \\
Neurologic & $13(26.0)$ \\
Respiratory & $13(26.0)$ \\
Cardiovascular & $9(18.0)$ \\
Endocrine & $3(6.0)$ \\
Tobacco use & $5(10.0)$ \\
None & $84.1(46.8-169.1)$ \\
Starting weight $(\mathrm{kg})$ & $30.8(16.1-56.6)$ \\
Starting BMl (kg/m $\left.{ }^{2}\right)$ & $33.0(17.8-56.6)$ \\
Female & $21.5(16.1-33.6)$ \\
Male &
\end{tabular}

Values are presented as number (\%) or median (range). GERD, gastroesophageal reflux disease; BMI, body mass index. ${ }^{\mathrm{a}}$ Median household income obtained from United States Census data.

\section{RESULTS}

\section{Demographic and clinical data}

Patients' demographic information and medical histories are presented in Table 1. The majority was female (68.0\%) and black (86.0\%). The median age at the time of consent was 47 years (range: 22-84 years) and twenty-eight patients (56.0\%) were 45 years or older. A significant proportion of patients had Medicaid $(42.0 \%)$ and the overall estimated median household income was approximately $\$ 31,000$. By contrast, the median household income across the state of Ohio is over $\$ 56,000$ [23]. The median number of active medications was six (range: 0-21). Patients had a variety of different medical comorbidities, the most common being hypertension, GERD, and neurologic/psychiatric conditions, such as depression or anxiety. A minority of patients were otherwise healthy with no known medical history (10.0\%). One patient had a history of thyroid cancer; however, no patients in this trial were undergoing active treatment for cancer.

Of note, one patient had bariatric surgery several years prior to trial initiation, but was not actively trying to lose further weight during the 26-week period. On review of patient histories and after a discussion with their primary care physician (co-author GR), two patients were attempting to lose weight through diet and exercise. The intent-to-study population ( $\mathrm{n}$ $=50$ ) was primarily analyzed, although five enrolled patients did not participate. One patient passed away shortly after enrolling and four others (8.0\%) failed to record any weights.

\section{Summary of patient-centered weight tracking compliance}

In the intent-to-study analysis, out of 1,300 possible individual recorded weights (50 patients, 26 weeks), 958 (73.7\%) were

Table 2. Summary of patient response rates in the weight tracking feasibility study, University Hospitals Cleveland Medical Center Primary Care Clinic, July 2019 to January 2020

\begin{tabular}{lcc}
\hline \multicolumn{1}{c}{ Characteristic } & All patient & $\begin{array}{c}\text { Participating } \\
\text { patient }\end{array}$ \\
\hline No. of patients & 50 & $45(90.0)$ \\
Total responses possible & 1,300 & 1,170 \\
Total responses recorded & $958(73.7)$ & $958(81.9)$ \\
$\quad$ First half responses & $505(77.7)$ & $505(86.3)$ \\
$\quad$ (weeks 1-13) & & \\
$\quad$ Second half responses & $453(69.7)$ & $453(77.4)$ \\
$\quad$ (weeks 14-26) & & \\
No. of responses recorded & & \\
$\geq 1(3.8 \%$ of total) & $45(90.0)$ & $45(100)$ \\
$\geq 13(50.0 \%$ of total) & $39(78.0)$ & $39(86.7)$ \\
$\geq 24(92.3 \%$ of total) & $26(52.0)$ & $26(57.8)$ \\
$\quad 26(100.0 \%$ of total) & $19(38.0)$ & $19(42.2)$ \\
Median no. of responses & $24(0-26)$ & $25(5-26)$ \\
Median response rate $(\%)$ & $92.3(0-100)$ & $96.2(20.2-100)$ \\
\hline
\end{tabular}

Values are presented as number only, number (\%), or median (range). 
recorded (Table 2). There was no difference in the response rate between the first half of the trial (weeks 1-13) and the second half (weeks $14-26$ ) (response rate: $77.7 \%$ vs. $69.7 \%$, $P=0.53$ ), reflecting good patient compliance. The median number of responses was 24 (out of a possible 26), which equates to a median individual response rate of $92.3 \%$. Twenty patients $(40.0 \%)$ recorded all 26 weights during the trial and $78.0 \%$ of patients recorded at least half of the possible weights (Fig. 2). The most common number of weights recorded, by far, was all of the possible weights.

In the on-study analysis, excluding five patients who were consented but did not participate, $81.9 \%$ of the possible 1,170 weight measurements were recorded. The median number of responses in this subgroup was 25 , or $96.2 \%$ of the possible responses (i.e., half of participants recorded $96.2 \%$ of the possible weights). All possible weights were recorded in $42.2 \%$ of participants and $86.7 \%$ recorded at least half of the possible weights.

A subgroup analysis was performed for patients 45 years and older ( $n=28,56.0 \%$ of total participants), since this is the demographic group that ultimately would be targeted for this cancer screening practice, based on increased cancer risk. Results are summarized in Table 3. Compliance to the weight tracking strategy over time was even higher in this older subgroup, as compared to the larger patient cohort. Similar to the full cohort, $72.7 \%$ of all possible weights were recorded in the intent-to-study cohort, and there was no difference in the response rate between the first and second half of the trial ( $76.4 \%$ vs. $71.7 \%, P=0.71)$. Over half of the patients greater than 45 years old recorded $96.2 \%$ of the possible weights. Notably, the two eldest patients in this trial (84 and 76 years old, both with multiple medical comorbidities) each recorded $100 \%$ of all possible weights.

\section{Summary of weight changes}

Although this is a feasibility study, we also determined if

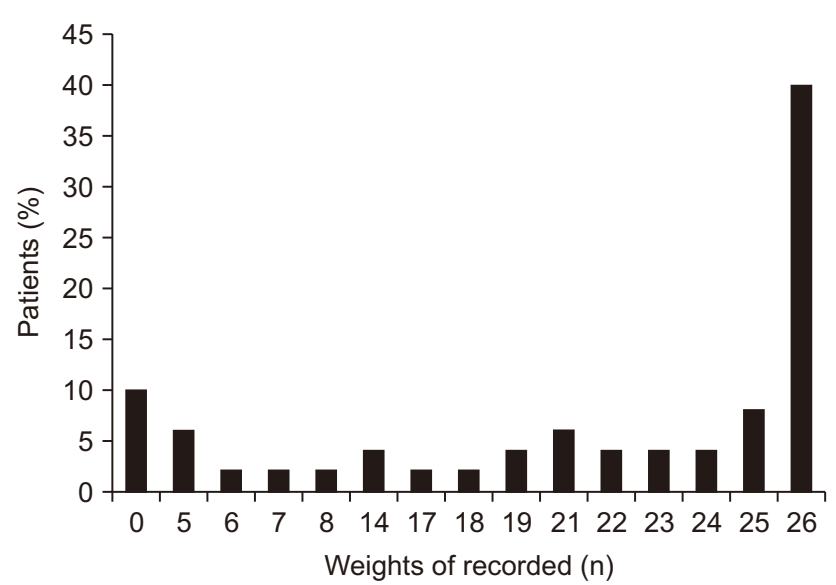

Figure 2. Frequency of weights recorded in the weight tracking feasibility study, University Hospitals Cleveland Medical Center Primary Care Clinic, July 2019 to January 2020. weight changes could be detected in this small sample of patients. Weight trends during the 26-week period across the intent-to-study cohort are summarized in Figure 3. A scatter plot of each patient's individual recorded weights is shown in Figure S1. Weight trends over the six-month study period are presented in greater detail in Table 4. For all patients, the maximum percent weight gain from baseline was $25.0 \%$, the maximum percent loss was $16.1 \%$, and the median weight change for all responses was $0 \%$. Twenty-eight patients $(56.0 \%)$ had stable weights with only minor fluctuations. Eleven patients $(22.0 \%)$ gained over $5 \%$ from their baseline body weight. Of those who experienced weight gain, seven $(14.0 \%$ of all patients) had persistent weight gain and four

Table 3. Summary of patient response rates, at least 45 years old, in the weight tracking feasibility study, University Hospitals Cleveland Medical Center Primary Care Clinic, July 2019 to January 2020

\begin{tabular}{lcc}
\hline \multicolumn{1}{c}{ Characteristic } & All patient & $\begin{array}{c}\text { Participating } \\
\text { patient }\end{array}$ \\
\hline No. of patients & $28(56.0)$ & $24(48.0)$ \\
Total responses possible & 728 & 624 \\
Total responses recorded & $529(72.7)$ & $529(84.8)$ \\
$\quad$ First half responses & $278(76.4)$ & $278(89.1)$ \\
$\quad$ (weeks $1-13)$ & & \\
$\quad$ Second half responses & $251(71.7)$ & $251(80.4)$ \\
$\quad$ (weeks $14-26)$ & & \\
No. of responses recorded & & \\
$\geq 1(3.8 \%$ of total) & $24(85.7)$ & $24(100)$ \\
$\geq 13(50.0 \%$ of total) & $21(75.0)$ & $21(87.5)$ \\
$\geq 25(96.2 \%$ of total) & $15(53.6)$ & $15(62.5)$ \\
$26(100.0 \%$ of total) & $12(42.9)$ & $12(50.0)$ \\
Median no. of responses & $25(0-26)$ & $25.5(5-26)$ \\
Median response rate & $96.2(0-100)$ & $100(20.2-100)$ \\
\hline
\end{tabular}

Values are presented as number (\%), number only, or median (range).

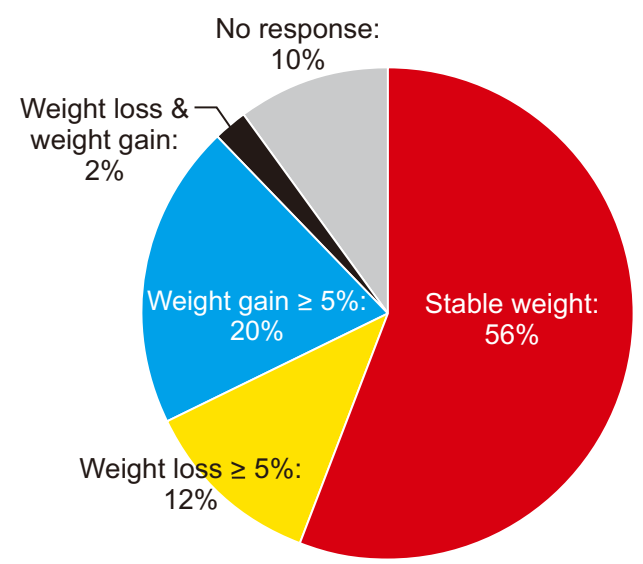

Figure 3. Summary of study population weight changes in the weight tracking feasibility study, University Hospitals Cleveland Medical Center Primary Care Clinic, July 2019 to January 2020 (intent-to-study, $\mathrm{n}=\mathbf{5 0}$ ). 
Table 4. Patient weight trends over the 26-week weight tracking feasibility study, University Hospitals Cleveland Medical Center Primary Care Clinic, July 2019 to January 2020

\begin{tabular}{lcc}
\hline \multicolumn{1}{c}{ Characteristic } & All patient & $\geq 45$ years old \\
\hline No. of patients & 50 & 28 \\
Weight change (\%) & $0(16.1$ [loss]-25.0 [gain]) & $0(11.8$ [loss]-25.0 [gain]) \\
No. with $\geq 5 \%$ weight loss $(\%)$ & $7(14.0)$ & $4(14.3)$ \\
Normalization of weight loss & $3(6.0)$ & $2(7.1)$ \\
Unintentional weight loss & $2(4.0)$ & $1(3.6)$ \\
Intentional weight loss & $2(4.0)$ & $1(3.6)$ \\
Number with $\geq 5 \%$ weight gain & $11(22.0)$ & $3(25.0)$ \\
Normalization of weight gain & $4(8.0)$ & $4(10.7)$ \\
Persistent weight gain & $7(14.0)$ & $17.3)$ \\
No. with stable weights & $28(56.0)$ & $17(60.7)$ \\
\hline
\end{tabular}

The entire intent-to-study cohort and the intent-to-study subgroup including patients at least 45 years of age are presented. Values are presented as number only, median (range), or number (\%).

(8.0\%) experienced normalization of their weight. One patient gained weight ( 63.2 to $66.8 \mathrm{~kg}, 5.4 \%$ gain from baseline) and then lost weight (most recent weight $58.2 \mathrm{~kg}, 7.9 \%$ loss from baseline) within the study period.

Seven patients $(14.0 \%)$ lost greater than $5 \%$ body weight from their baseline. Out of these patients, four $(8.0 \%$ of all patients) had persistent weight loss, while three $(6.0 \%)$ experienced a return towards baseline (transient weight loss). In the group of four patients who had persistent weight loss greater than $5 \%$, two of the patients were actively trying to lose weight (intentional weight loss). However, two patients (4.0\%) experienced UWL during this 26 -week period. One patient was younger than 45 years old and was referred to a colorectal surgeon to further evaluate gastrointestinal symptoms. The second patient is under cautions follow-up to monitor for new symptoms that might explain the UWL.

\section{DISCUSSION}

Early detection of cancer has multiple anticipated benefits. First, quicker time to diagnosis and treatment will likely downstage many patients, resulting in improved cancer-specific survival. Mathematical modeling in pancreatic cancer based on mutation rates estimates that a 6-18-month lead-time could translate to a transition from localized to systemic disease [24]. The five-year survival rates for localized, regional, and metastatic lung cancer are $57 \%, 31 \%$, and $5 \%$, respectively [17]. Relative survival patterns across cancer stages mirror this trend in diverse tumor types [17]. A second benefit to early detection is that cancer treatments have a greater impact. One of the most important predictors of chemotherapy success relates to the extent of disease [25-28]. In addition, health and performance status declines in cancer patients quickly, often over just a few months. Patients with advanced disease are ultimately unable to tolerate treatment side effects. Advanced cancer often becomes resistant to available therapies $[29,30]$. Thus, it stands to reason that earlier detection of cancer by just a few months could have a multiplier effect on patient survival.

Cancer has been linked to weight loss for decades [11], but this early warning sign or symptom has not been considered systematically as an actual early detection tool. Several features of UWL are especially attractive for this purpose. UWL is common among most malignancies [11]. Therefore, UWL screening could be the first universal early detection method. In contrast to some traditional cancer detection strategies (mammogram, prostate-specific antigen), there are essentially no false positives [31,32]. Although an underlying etiology cannot be determined in approximately $20 \%$ of UWL cases [1-9], most instances are believed to result from some abnormal pathology (cancer or a benign disease process). Thus, all cases of UWL should be investigated. The test may be performed at home, and at frequent intervals. This feature contrasts with traditional, and investigational, cancer detection strategies. Colonoscopies, mammograms, Papanicolaou smears, and liquid biopsies, are all performed at intervals of a year or more [33-35].

Home-based, daily or weekly health monitoring technologies are already performed commonly by patients. Examples include finger-stick glucose and blood pressure measurements [36-38]. We hypothesized that patients would also be comfortable and willing to participate in weight weekly tracking [39]. Similarly, in an era where telehealth is becoming increasingly common [40], reliance on home-based body weight measurements may become more prevalent. Finally, the low cost of patient-centered weight tracking is highly appealing compared to other cancer detection methods. There has been heavy investment in more sophisticated early detection strategies like blood-based molecular interrogation technologies. Liquid biopsies would be administered every few months (if not yearly) and are comparatively expensive [41]. By contrast, weight tracking was performed in the present study using a $\$ 10$ bathroom scale.

Herein, we report the results of the first-ever prospective trial of weight tracking, with the eventual goal to detect underlying and undiagnosed pathology associated with UWL. 
Patient-centered weight tracking is highly feasible at weekly intervals, performed over the course of a six-month study period. We believe these data are generalizable to patients atrisk for developing cancer. More than half the patients in the present study were over 45 years old and patients enrolled in this study were generally from neighborhoods with a relatively depressed socioeconomic status. Furthermore, this weight tracking strategy detected weight fluctuations, including expected weight loss from patients actively trying to lose weight. The incidence of UWL seen in this study was $4 \%$ over the six-month period, or roughly $8 \%$ extrapolated over a year. This rate is consistent with prior projections of UWL in similar age groups [1-9].

This prospective trial has several limitations. These include the small sample size. We are not able to verify that all reported weights were accurate. We did not validate the data with actual photos or use raw data from the scales. However, the fact that patients with "stable weight" experienced minor weight fluctuations suggests that patients were not simply entering the same weight, but rather entering a unique measurement each time. Also, patients in the study received a small amount of monetary compensation.

\section{Future studies}

These findings support future attempts to improve weight tracking and scale to larger populations, with a goal to detect occult malignancies. Smart-scale technology will enable the use of raw weight data, improve patient compliance, and minimize human error. Telephone applications can automate reminders and improve compliance. Data may be integrated into the electronic medical record in real time, analyzed, and generate alerts when UWL occurs. We hope to initiate a large-scale prospective weight tracking study (performed weekly) in thousands of patients to test this approach as an early cancer detection strategy. If we conservatively estimate a $5 \%$ yearly incidence of UWL, patient-centered weight tracking may detect roughly 100 cases of UWL in a study of 1,000 patients, over two years. Based on historical rates of malignancy in cohorts with UWL ( $20 \%$ of cases), 20 of these patients are expected to harbor an occult malignancy. A diagnostic path can then be designed and applied to these patients as part of a targeted work-up. Relevant tests may include a patient history, physical examination, lab work, endoscopy, and cross-sectional imaging.

In conclusion, this study shows that patient weight can be reliably tracked using simple and inexpensive tools. The incidence of UWL is approximately $4 \%$ over a six-month period. These preliminary data provide a foundation for a larger scale clinical trial to evaluate the utility of patient-centered weight tracking to detect occult malignancies and other diseases.

\section{ACKNOWLEDGMENTS}

This study was funded intramurally at University Hospitals
Cleveland Medical Center by the University Hospitals Ventures START (Stimulation of Teams, and Activation of Resources and Tools) Fund, with support and guidance from the University Hospitals Cleveland Medical Center Research Health Analytics and Informatics Program.

The study team would like to acknowledge Devblock for their assistance and innovation in design of this project. Please contact Benjamin Liu (ben@devblock.net) for more information on Devblock Technologies. We also would like to acknowledge the patients for their willingness to participate in this study, Sue Ables for assisting in preparing the scales for patients, and Ryan Jastromb for assisting in delivering enrollment funds to patients.

\section{CONFLICTS OF INTEREST}

No potential conflicts of interest were disclosed.

\section{SUPPLEMENTARY MATERIALS}

Supplementary materials can be found via https://doi. org/10.15430/JCP.2020.25.3.181.

\section{ORCID}

Jonathan J. Hue, https://orcid.org/0000-0002-1608-8874

Sarah C. Markt, https://orcid.org/0000-0003-2873-0358

Goutham Rao, https://orcid.org/0000-0001-7739-9353

Jordan M. Winter, https://orcid.org/0000-0002-5200-6557

\section{REFERENCES}

1. Wong CJ. Involuntary weight loss. Med Clin North Am 2014;98:625-43.

2. Marton KI, Sox HC Jr, Krupp JR. Involuntary weight loss: diagnostic and prognostic significance. Ann Intern Med 1981;95:568-74.

3. Rabinovitz M, Pitlik SD, Leifer M, Garty M, Rosenfeld JB. Unintentional weight loss. A retrospective analysis of 154 cases. Arch Intern Med 1986;146:186-7.

4. Lankisch P, Gerzmann M, Gerzmann JF, Lehnick D. Unintentional weight loss: diagnosis and prognosis. The first prospective follow-up study from a secondary referral centre. J Intern Med 2001;249:41-6.

5. Thompson MP, Morris LK. Unexplained weight loss in the ambulatory elderly. J Am Geriatr Soc 1991;39:497-500.

6. Bilbao-Garay J, Barba R, Losa-García JE, Martín H, García de Casasola G, Castilla V, et al. Assessing clinical probability of organic disease in patients with involuntary weight loss: a simple score. Eur J Intern Med 2002;13:240-5.

7. Metalidis C, Knockaert DC, Bobbaers H, Vanderschueren S. Involuntary weight loss. Does a negative baseline evaluation provide adequate reassurance? Eur J Intern Med 2008;19:3459. 
8. Wu JM, Lin MH, Peng LN, Chen LK, Hwang SJ. Evaluating diagnostic strategy of older patients with unexplained unintentional body weight loss: a hospital-based study. Arch Gerontol Geriatr 2011;53:e51-4.

9. Chen SP, Peng LN, Lin MH, Lai HY, Hwang SJ, Chen LK. Evaluating probability of cancer among older people with unexplained, unintentional weight loss. Arch Gerontol Geriatr 2010;50 Suppl 1:S27-9.

10. Tan BH, Fearon KC. Cachexia: prevalence and impact in medicine. Curr Opin Clin Nutr Metab Care 2008;11:400-7.

11. Dewys WD, Begg C, Lavin PT, Band PR, Bennett JM, Bertino JR, et al. Prognostic effect of weight loss prior to chemotherapy in cancer patients. Eastern Cooperative Oncology Group. Am J Med 1980;69:491-7.

12. Olson SH, Xu Y, Herzog K, Saldia A, DeFilippis EM, Li P, et al. Weight loss, diabetes, fatigue, and depression preceding pancreatic cancer. Pancreas 2016;45:986-91.

13. Morel H, Raynard B, d'Arlhac M, Hauss PA, Lecuyer E, Oliviero $\mathrm{G}$, et al. Prediagnosis weight loss, a stronger factor than BMI, to predict survival in patients with lung cancer. Lung Cancer 2018;126:55-63.

14. van Zutphen M, Geelen A, Boshuizen HC, Winkels RM, Geijsen AJMR, Wesselink E, et al. Pre-to-post diagnosis weight trajectories in colorectal cancer patients with non-metastatic disease. Support Care Cancer 2019;27:1541-9.

15. Li H, Li Y, Liu Y, Huang D, Bai M, Ge S, et al. The incidence and impact of weight loss with cachexia in gastric cancer patients. J Clin Oncol 2015;33(15_suppl):e20644.

16. El-Kareh R, Pazo V, Wright A, Schiff GD. Losing weights: failure to recognize and act on weight loss documented in an electronic health record. J Innov Health Inform 2015;22:316-22.

17. Siegel RL, Miller KD, Jemal A. Cancer statistics, 2020. CA Cancer J Clin 2020;70:7-30.

18. National Cancer Institute. Cancer Statistics. https://www.cancer. gov/about-cancer/understanding/statistics. Accessed July 2, 2019.

19. American Cancer Society. Facts \& figures 2019: US cancer death rate has dropped $27 \%$ in 25 years. https://www.cancer.org/ latest-news/facts-and-figures-2019.html. Accessed July 2, 2019.

20. National Cancer Institute: Surveillance, Epidemiology, and End Results Program. Cancer stat facts: cancer of any site. https:// seer.cancer.gov/statfacts/html/all.html. Accessed July 1, 2020.

21. National Cancer Institute. Budget fact book for fiscal year 2015. Bethesda, MD, National Cancer Institute, 2017.

22. Clinical OMICs. 10 Liquid biopsy companies in the money. https://www.clinicalomics.com/topics/oncology/10-liquid-biopsycompanies-in-the-money/. Accessed May 12, 2020.

23. United States Census Bureau. Income data tables. https://www. census.gov/topics/income-poverty/income/data/tables.html. Accessed April 26, 2020.

24. Yachida S, Jones S, Bozic I, Antal T, Leary R, Fu B, et al. Distant metastasis occurs late during the genetic evolution of pancreatic cancer. Nature 2010;467:1114-7.

25. Conroy T, Hammel P, Hebbar M, Ben Abdelghani M, Wei
AC, Raoul JL, et al.; Canadian Cancer Trials Group and the Unicancer-GI-PRODIGE Group. FOLFIRINOX or gemcitabine as adjuvant therapy for pancreatic cancer. $\mathrm{N}$ Engl $\mathrm{J}$ Med 2018;379:2395-406.

26. Conroy T, Desseigne F, Ychou M, Bouché O, Guimbaud R, Bécouarn Y, et al.; Groupe Tumeurs Digestives of Unicancer; PRODIGE Intergroup. FOLFIRINOX versus gemcitabine for metastatic pancreatic cancer. N Engl J Med 2011;364:1817-25.

27. Wilkinson NW, Yothers G, Lopa S, Costantino JP, Petrelli NJ, Wolmark N. Long-term survival results of surgery alone versus surgery plus 5-fluorouracil and leucovorin for stage II and stage III colon cancer: pooled analysis of NSABP C-01 through C-05. A baseline from which to compare modern adjuvant trials. Ann Surg Oncol 2010;17:959-66.

28. Scheithauer W, Rosen H, Kornek GV, Sebesta C, Depisch D. Randomised comparison of combination chemotherapy plus supportive care with supportive care alone in patients with metastatic colorectal cancer. BMJ 1993;306:752-5.

29. Kindler HL, Niedzwiecki D, Hollis D, Sutherland S, Schrag D, Hurwitz H, et al. Gemcitabine plus bevacizumab compared with gemcitabine plus placebo in patients with advanced pancreatic cancer: phase III trial of the Cancer and Leukemia Group B (CALGB 80303). J Clin Oncol 2010;28:3617-22.

30. Kalser MH, Ellenberg SS. Pancreatic cancer. Adjuvant combined radiation and chemotherapy following curative resection. Arch Surg 1985;120:899-903.

31. Tosteson AN, Fryback DG, Hammond CS, Hanna LG, Grove MR, Brown M, et al. Consequences of false-positive screening mammograms. JAMA Intern Med 2014;174:954-61.

32. Bernal-Soriano MC, Parker LA, López-Garrigos M, HernándezAguado I, Caballero-Romeu JP, Gómez-Pérez L, et al. Factors associated with false negative and false positive results of prostate-specific antigen (PSA) and the impact on patient health: cohort study protocol. Medicine (Baltimore) 2019;98:e17451.

33. US Preventive Services Task Force, Bibbins-Domingo K, Grossman DC, Curry SJ, Davidson KW, Epling JW Jr, et al. Screening for colorectal cancer: US Preventive Services Task Force recommendation statement. JAMA 2016;315:2564-75.

34. US Preventive Services Task Force, Curry SJ, Krist AH, Owens DK, Barry MJ, Caughey AB, et al. Screening for cervical cancer: US Preventive Services Task Force recommendation statement. JAMA 2018;320:674-86

35. Siu AL; U.S. Preventive Services Task Force. Screening for breast cancer: U.S. Preventive Services Task Force recommendation statement. Ann Intern Med 2016;164:279-96.

36. George J, MacDonald T. Home blood pressure monitoring. Eur Cardiol 2015;10:95-101.

37. Hodgkinson J, Mant J, Martin U, Guo B, Hobbs FD, Deeks $\mathrm{JJ}$, et al. Relative effectiveness of clinic and home blood pressure monitoring compared with ambulatory blood pressure monitoring in diagnosis of hypertension: systematic review. BMJ 2011;342:d3621.

38. Olansky L, Kennedy L. Finger-stick glucose monitoring: issues of accuracy and specificity. Diabetes Care 2010;33:948-9. 
Hue et al.

39. Ingels JS, Misra R, Stewart J, Lucke-Wold B, Shawley-Brzoska $\mathrm{S}$. The effect of adherence to dietary tracking on weight loss: using HLM to model weight loss over time. J Diabetes Res 2017;2017:6951495.

40. Hollander JE, Carr BG. Virtually perfect? Telemedicine for covid-19. N Engl J Med 2020;382:1679-81.

41. Duffy MJ, Sturgeon CM, Sölétormos G, Barak V, Molina R, Hayes DF, et al. Validation of new cancer biomarkers: a position statement from the European group on tumor markers. Clin Chem 2015;61:809-20. 